\title{
Truncating ARL6IP1 variant as the genetic cause of fatal complicated hereditary spastic paraplegia
}

\author{
Salma M. Wakil ${ }^{1,2^{*}}$, Safa Alhissi ${ }^{1}$, Haya Al Dossari ${ }^{1}$, Ayesha Alqahtani ${ }^{1}$, Sherin Shibin ${ }^{1}$, Brahim T. Melaiki ${ }^{4}$,
} Josef Finsterer ${ }^{3}$, Amal Al-Hashem ${ }^{4}$, Saeed Bohlega ${ }^{5}$ and Anas M. Alazami ${ }^{1,2}$

\begin{abstract}
Background: Mutations in ARL6IP1, which encodes a tetraspan membrane protein localized to the endoplasmic reticulum (ER), have been recently described in a large family with a complicated form of hereditary spastic paraplegia (HSP).

Case presentation: We sought to expand the HSP phenotype associated with ARL6IP1 variants by examining a Saudi kindred with a clinically more severe presentation, which resulted in spontaneous neonatal death of both affected siblings. Clinical features encompassed not only spastic paraplegia but also developmental delay, microcephaly, cerebral atrophy, periventricular leukoencephalopathy, hypotonia, seizures, spasticity, jejunal stricture, gastrointestinal reflux, neuropathy, dysmorphism and respiratory distress. We performed clinical assessment and radiological studies of this family, in addition to homozygosity mapping and whole exome sequencing (WES) to identify the disease-associated variant. Homozygosity mapping localized the causative gene to a region on chromosome 16 harboring ARL6IP1. WES of the index case identified the homoallelic nonsense variant, c.112C > T in ARL6IP1 that segregated with the phenotype and was predicted to result in loss of the protein. Allelic expression analysis of the parents demonstrated downward pressure on the mutant allele, suggestive of nonsense-mediated decay.
\end{abstract}

Conclusions: Our report shows that the phenotype associated with ARL6IP1 variants may be broader and more acute than so far reported and identifies fatal HSP as the severe end of the phenotypic spectrum of ARLGIP1 variants.

Keywords: Hereditary spastic paraplegia, Autosomal recessive, Whole exome sequencing, ADP ribosylation factor like GTPase 6 interacting protein 1

\section{Background}

Hereditary spastic paraplegia (HSP) represents a highly heterogeneous group of complex neurological disorders involving multiple loci and causative genes. HSP is clinically characterized by progressive weakness and spasticity, predominantly in the lower limbs, and is further subdivided into a pure and a complex form [1]. OMIM has assigned identifiers to 79 genes or loci that have

\footnotetext{
* Correspondence: smajid@kfshrc.edu.sa

'Department of Genetics, Research Centre, King Faisal Specialist Hospital \& Research Centre, MBC-03, P.O. Box 3354, Riyadh 11211, Saudi Arabia

${ }^{2}$ Saudi Human Genome Program, King Abdulaziz City for Science and

Technology, Riyadh, Saudi Arabia

Full list of author information is available at the end of the article
}

been linked to spastic paraplegia (SPG1-SPG79, in order of their discovery), although this list is not comprehensive and continues to expand [2-4]. All modes of inheritance have been reported.

The human ADP ribosylation factor like GTPase 6 interacting protein $1(A R L 6 I P 1)$ gene, situated at locus $16 \mathrm{p} 12.3$ encodes a tetraspan membrane protein that regulates intracellular trafficking pathways in the endoplasmic reticulum (ER) membrane [5]. ARL6IP1 acts as an anti-apoptotic protein specific to multicellular organisms, and is a potential player in shaping the ER tubules in mammalian cells. In neurons it has been associated with regulation of glutamate, a major excitatory 
neurotransmitter in excitatory synapse [6]. In drosophila, knockdown of the gene leads to progressive motor deficit [7].

Recently an ARL6IP1 variant has been reported in a patient with spastic paraplegia, motor and sensory polyneuropathy and acromutilation [8]. Here, we report a lethal homoallelic truncating variant in ARL6IP1 manifesting with classical features of HSP and additionally with dysmorphic features, developmental delay, microcephaly, neuropathy, leukoencephalopathy, partial agenesis of corpus callosum, seizures, high grade gastroesophageal reflux disease, and respiratory distress in two affected members of a consanguineous Saudi family. This presentation of features expands the phenotypic spectrum of ARL6IP1-associated HSP.

CARE guidelines were followed in this report.

\section{Case presentation}

Patient 1 was a 27 month old girl (Fig. 1a) born at full term pregnancy but too small for gestational age: her birth weight was $2.12 \mathrm{~kg}$ ( $<10$ th centile), length $48 \mathrm{~cm}$, and head circumference $31 \mathrm{~cm}(<10$ th centile). She presented with failure to thrive, dysmorphic features including microcephaly ( $\mathrm{z}$ score: -2.4), severe lumbar hyperlordosis, limited hip abduction and neuropathy. Shortly after birth the patient developed respiratory distress requiring intubation. Several attempts of extubation were frustrating and the patient underwent tracheostomy. She had episodes of seizures controlled by phenobarbital. Since EEG did not show epileptiform discharges, phenobarbital was discounted without recurrence of seizures. Cerebral MRI revealed atrophy with dilated ventricles and enlarged subarachnoid space, extensive periventricular leukoencephalopathy, and partial agenesis of the corpus callosum (Fig. 2). Echocardiography revealed a patent foramen ovale and a patent ductus arteriosus. At age 1 month she developed gastrointestinal reflux due to intestinal obstruction secondary to jejunal stricture, managed by resection of the stricture and end-to-end anastomosis. Gastroscopy revealed a high-grade reflux disease. The index patient continued to have severe delay in all developmental aspects, did not gain any milestones, and continued to be ventilation-dependent. The further course was complicated by chronic lung disease. At 24 months she experienced an episode of apnea, and died from cardiac arrest at age 28 months. The karyotype was XX.

Patient 2, the brother of patient 1 (Fig. 1a), was delivered by elective Caesarian section at gestational week 37 because of placenta previa. He was admitted to the NICU because of hypotonia and respiratory distress. On examination, his birth weight was $2.3 \mathrm{~kg}$ (between the

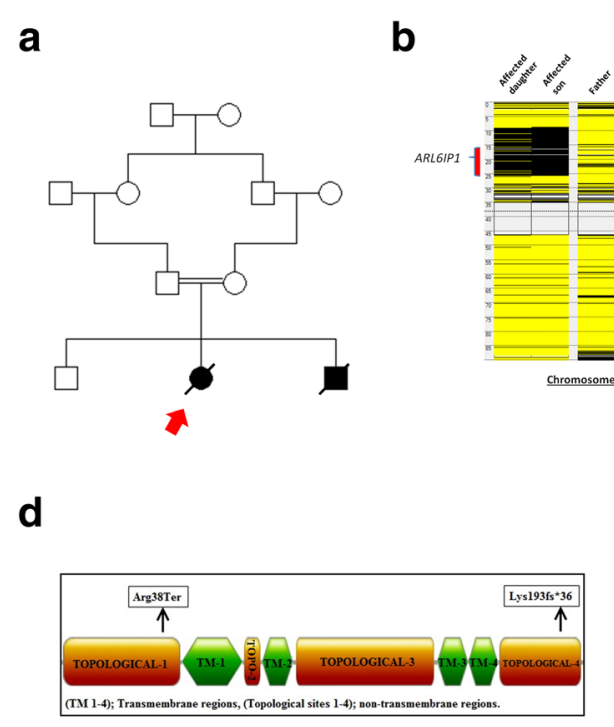

C

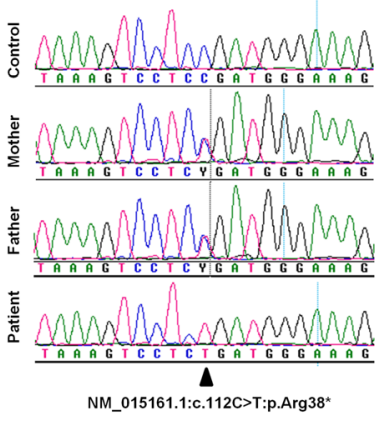

e

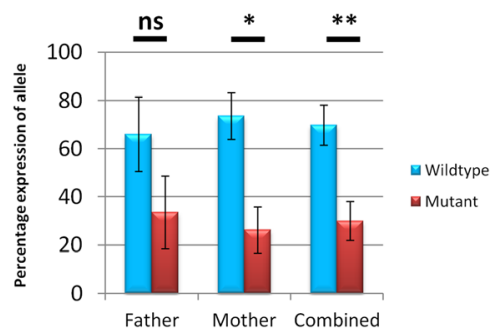

Fig. 1 a. Pedigree of the family from this study. The index case (whose DNA was subjected to WES) is indicated with an arrow. $\mathbf{b}$ AutoSNPa software showing block of homozygosity (black) of family on chromosome 16 encompassing the ARL6IP1 gene. Yellow regions indicate heterozygous SNPs. c Sequence electropherogram traces showing the mutation C.112C > T in one patient compared with both parents and a normal control. d Gene domains of ARL6IP1 showing mutations reported thus far. e Analysis of the wildtype vs mutant allelic expression for both parents, based on three independent low-cycle RT-PCR reactions cloned into TOPO vector. Asterisks indicate significance levels $\left({ }^{*} p<0.05\right.$, ${ }^{* *} p<$ $0.01, \mathrm{~ns}=$ not significant) 


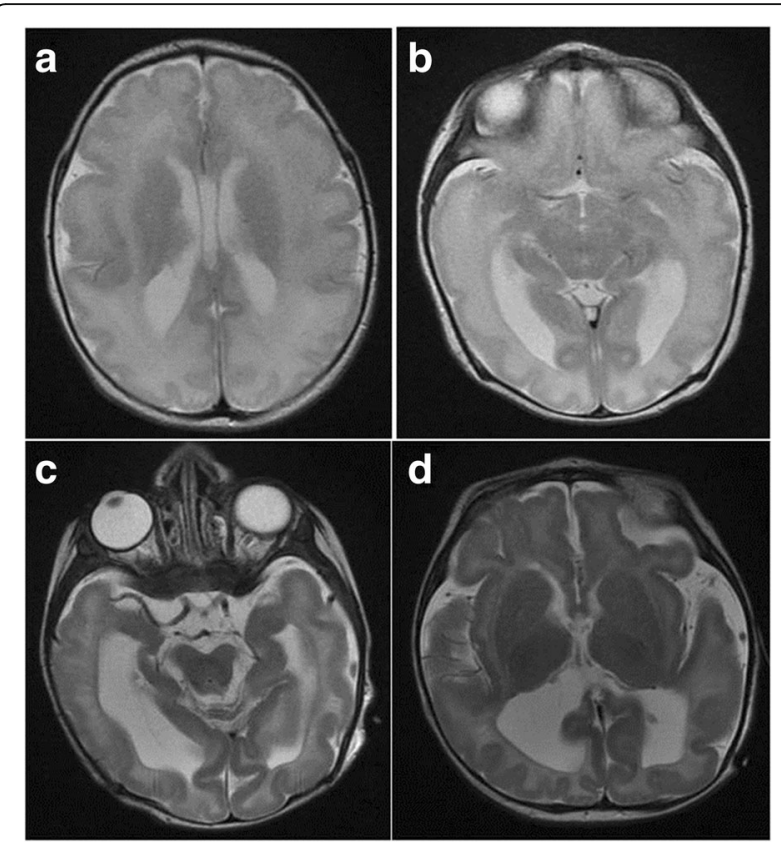

Fig. 2 T2-weighted axial images of the cerebrum showing diffuse hyperintensity and atrophy of white matter $(\mathbf{a}, \mathbf{b}, \mathbf{c}, \mathbf{d})$, dilated ventricles $(\mathbf{b}, \mathbf{c}, \mathbf{d})$, enlarged subarachnoid space $(\mathbf{c}, \mathbf{d})$, and partial agenesis of the corpus callosum (absence of splenium) (d)

10th and 50th percentile). Length was $48 \mathrm{~cm}$ (at the 50th percentile), and he had microcephaly (head circumference $32 \mathrm{~cm}$ at 2.6 centile, $Z$ score - 1.9). He had overriding sutures, small fontanels, prominent nasal bridge, thin lips, microcephaly plagiocephaly, retrognathia, high arched palate, long hands, long fingers, hyper-extendable joints, redundant skin, large feet, severe head lag, absent deep tendon reflexes, and generalized hypotonia. Later in the course, he developed spasticity. Cerebral $1.5 \mathrm{~T}$ MRI was normal as well as metabolic screen (tandem MS, urine GCSM, ammonia, lactate, pyruvate, CK). Upon nerve conduction studies supramaximal stimulation did not evoke a motor response in any of the investigated nerves. Needle EMG revealed a neurogenic pattern. He required naso-gastric tube feeding and did not gain weight despite high caloric feeding. He required nasal cannula flow, HB-CPAP, and at age 52 days he was intubated. Extubation was frustrating several times. At times with progressive increase of oxygen demand he required high ventilator support. The further course was complicated by the development of pulmonary compromise, such as chronic lung disease and atelectasis on thoracic CT and ventilatorassociated pneumonia. A study of the upper gastrointestinal tract showed gastro-esophageal reflux disease (GERD) managed with anti-reflux formula and medications. The patient eventually died at age of 16 months. The karyotype was XY.
The parents were first-degree cousins, and the family history was positive for neonatal deaths of individuals with joint contractures who died from respiratory distress.

Initial genetic workup for both patients involved molecular karyotyping using Cytoscan HD (Affymetrix, Santa Clara, USA), which contains 2.6 million markers of which 750,000 are genotyping single nucleotide polymorphisms and the remainder are non-polymorphic probes for genome coverage. Data analysis, using Chromosome Analysis Suite version Cyto 2.0.0.195(r5758), indicated that all individuals' karyotypes were normal.

We next carried out homozygosity analysis via the Affymetrix Axiom array (Affymetrix, Santa Clara, CA, USA). All sets of homozygous stretches of SNPs were analyzed for shared runs of homozygosity (ROH) using autoSNPa (http://dna.leeds.ac.uk/autosnpa/), with a preset minimum $2 \mathrm{Mb}$ size limit. Utilizing the five available family members, homozygosity data revealed a single $\mathrm{ROH}$ on chromosome 16 that was exclusive to the two affected members of this family (Fig. 1b). These findings were further interrogated using WES. DNA from the index case was treated to obtain an Ion Proton AmpliSeq library, which then underwent emulsion PCR on an Ion OneTouch System. Templated Ion Sphere particles were enriched using Ion OneTouch ES (Life Technologies, Carlsbad, CA, USA). The template-positive Ion PI Ion Sphere particles were processed for sequencing on the Ion Proton instrument (Life Technologies, Carlsbad, CA, USA); reads were mapped to UCSC hg19 (http://genome. ucsc.edu/) and variants identified using the Ion Torrent pipeline (Life Technologies, Carlsbad, CA, USA).

WES resulted in (on average) $>100 x$ coverage totaling $46.2 \mathrm{Mb}$ of genomic sequence from the index case. The resultant variant caller file (VCF) was filtered as follows. Given that the parents were consanguineous and asymptomatic, and given the presence of strong family history and the similarity of clinical presentation between both affected, only homozygous variants were considered in our analyses. Additionally, variants were excluded if they exhibited a high minor allele frequency $(\mathrm{MAF}>0.01)$, were previously reported variants (present in dbSNP, 1000 genomes, 1500 Saudi exomes) or were present in 1200 chromosomes from the Saudi population. Only one homozygous variant survived the filtration process: ARL6IP1 (NM_015161.1: c.112C > T: p.Arg38*) (Fig. 1c). Sanger sequencing on an ABI 3730xl automated sequencer (Applied Biosystems, Foster City, CA) confirmed that the variant segregated with the disease state.

This variant causes an arginine residue to be changed into a stop codon at position 38 (p.Arg38*) in the topological domain of ARL6IP1 (Fig. 1d). Given the truncating nature of the variant we wanted to assess whether the affected alleles were undergoing nonsense-mediated decay (NMD). Since no patient RNA was available, we 


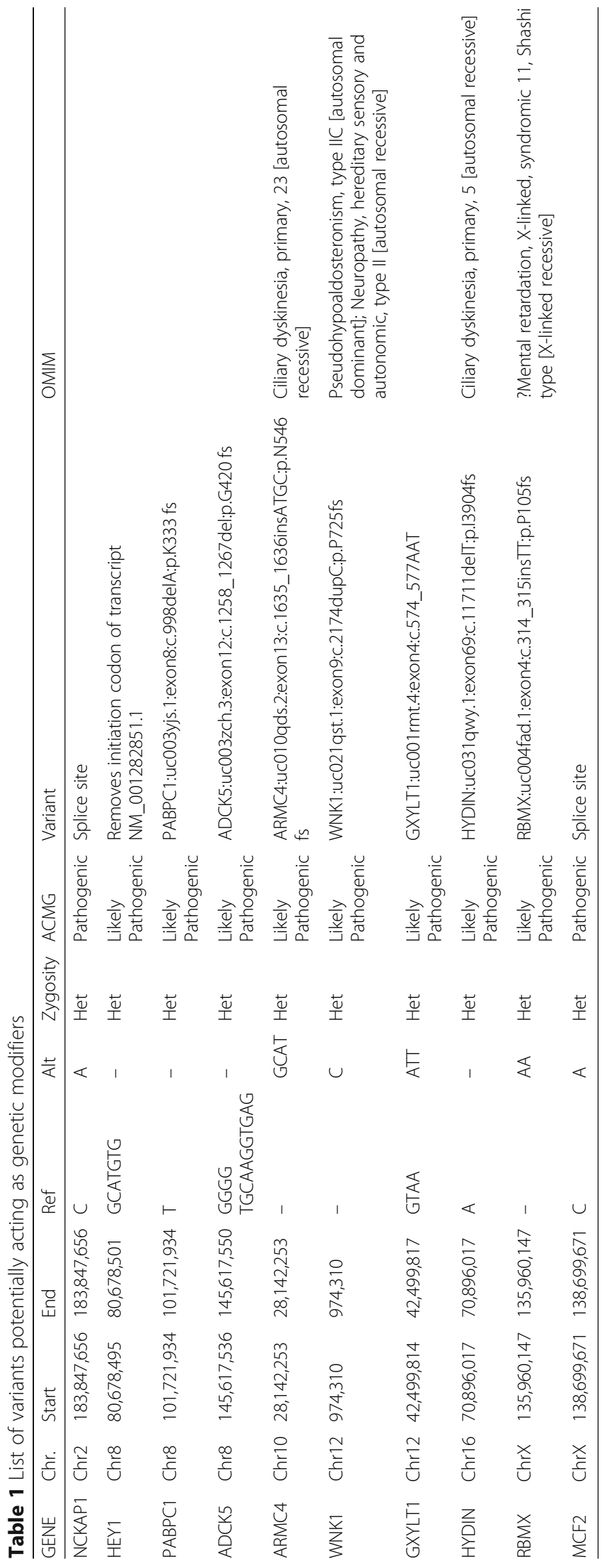


examined the expression ratios of the wild type versus mutant alleles in the two obligate carriers (the parents). Total RNA was extracted from Paxgene tubes (PreAnalytix, Switzerland) then converted to cDNA. Reverse transcriptase (RT) PCR primers (5' -AAGAACAGCTGCAAGGATGG-3' and 5'-GGTTGTGGACTTGTTGTC CC-3') were used to amplify a region incorporating the mutation site. The amplification was conducted for a low number of cycles (18-20) to ensure that the reaction did not reach saturation levels. RT-PCR products were then cloned into a TOPO vector (Thermo Fisher, Waltham MA, USA).

For each parent, 150 bacterial transformants (grown on antibiotic-coated agar plates) were isolated for subsequent study, based on three independent rounds of lowcycle-number RT-PCR and cloning. By employing twotailed unpaired Student's t-test, we identified a trend in the father of higher expression for the wild type allele, although it did not reach significance (defined as $p<$ 0.05) (Fig. 1e). However the results from the mother were significant $(p<0.03)$, and even more so when the data for both parents were combined $(p<0.01)$. This strongly suggested that RNA from the mutant allele was undergoing degradation due to NMD surveillance.

\section{Discussion and conclusions}

ARL6IP1 is an integral transmembrane protein and its main function lies in ER shaping. It contains hairpin loop domains that localize to smooth ER tubules $[5,9]$. ER-shaping proteins play an intrinsic role in the network of mitochondrial organization in motor neurons. Studies have shown the role of ARL6IP1 in elongation of axonal mitochondria, suggesting that loss of function variants in ER-shaping proteins disrupt mitochondrial network organization in motor neurons [7].

Homozygous variants in ARL6IP1 were previously reported in patients with spastic paraplegia, diffuse sensory and motor polyneuropathy and acromutilation. These patients had late onset of disease and were diagnosed at 14 months, and by 10 years they could walk with support but with unsteadiness and scissors gait. They had skeletal deformities (loss of terminal digits) but cognition was normal [10]. In another study a patient was born to second-cousin parents with reduced head circumference $(31.5 \mathrm{~cm})$, and presented with acromutilation and congenital insensitivity to pain with small fibers involvement as additional features [8]. Crawling began at 14 months and the patient was able to walk with support at 4.5 years of age. The variant reported here has been recently identified in another family [11]. However, the phenotype found in our two patients is more severe than all other reported ARL6IP1 patients to date. It is intriguing to speculate on the reasons why. Clinical workup for both affected did not point to any perinatal infections.
We cannot rule out confounding environmental factors or genetic modifiers. We note that there is a family history among relatives of skeletal dysplasia and early infantile death. A subset of variants from the WES data (filtered by zygosity and ACMG classification) are provided in Table 1 . Some of these may act as genetic modifiers, for example ARMC4 and HYDIN are both associated with autosomal recessive primary ciliary dyskinesia, and although the variants are heterozygous they may have played a role in the newborns' respiratory distress. Similarly the NCKAP1 and $R B M X$ variants may have exerted an effect on neuronal function.

Here we present a rare form of HSP characterized by cerebral abnormalities (failure to thrive, developmental delay, atrophy, leucoencephalopathy, generalized hypotonia, spasticity, partial agenesis of the corpus callosum), jejunal stricture, patent foramen ovale, patent ductus, distinct dysmorphic features, respiratory distress, culminating in respiratory insufficiency and neonatal death. Dysmorphic features included microcephaly, plagocephaly, overriding sutures, small fontanels, prominent nasal bridge, thin lips, retrognathia, high arched palate, long hands, long fingers, hyper-extendable joints, lumbosacral hyperlordosis, and large feet.

The presence of a rapidly progressive, complicated and fatal HSP broadens the clinical image associated with ARL6IP1 variants, outlining the severe end of the phenotypic spectrum for mutations in this gene.

\section{Abbreviations}

ER: Endoplasmic reticulum; GERD: Gastro-esophageal reflux disease; HSP: Hereditary spastic paraplegia; MAF: Minor allele frequency; RT: Reverse transcriptase; SPG: Spastic paraplegia gene; VCF: Variant caller file

\section{Acknowledgements}

We are grateful to all family members for their generous participation in this study. We thank the Sequencing and Genotyping Core Facilities of the Department of Genetics, Research Centre, King Faisal Specialist Hospital and Research Centre. We gratefully acknowledge the use of Saudi Human Genome Project resources, funded by the King Abdulaziz City for Science and Technology, Riyadh, Saudi Arabia.

\section{Authors' contributions}

SMW and AMA designed the study, analyzed data, and prepared the manuscript; SA performed experiments and tabulated data; HAD, AA and SS generated the genotyping data; JF participated in collating and writing the clinical data; AA-H \& BTM cared for patients and contributed clinical data; SB cared for patients and contributed and wrote up clinical data. All authors read and approved the final manuscript.

Funding

No external (non-institutional) funding was used for this study.

\section{Availability of data and materials}

Genotyping and whole exome sequencing data are available from the corresponding author on written request.

Ethics approval and consent to participate

All the subjects in this study were enrolled under an IRB approved by King Faisal Specialist Hospital \& Research Centre (RAC\# 2090011) with full informed written consent. 


\section{Consent for publication}

Although no personally identifiable information is presented in this study, written informed consent for publication was obtained from the parents/ legal guardians of the two infants reported in this study. Consent for publishing this report was also acquired from the Office of Research Affairs at King Faisal Specialist Hospital \& Research Centre (RAC Publication \# 2190035).

\section{Competing interests}

The authors declare that they have no competing interests.

\section{Author details}

'Department of Genetics, Research Centre, King Faisal Specialist Hospital \& Research Centre, MBC-03, P.O. Box 3354, Riyadh 11211, Saudi Arabia. ${ }^{2}$ Saudi Human Genome Program, King Abdulaziz City for Science and Technology, Riyadh, Saudi Arabia. ${ }^{3}$ Department of Neurology, Krankenanstalt Rudolfstiftung, Messerli Institute, Postfach 20, 1180 Vienna, Austria. ${ }^{4}$ Prince Sultan Riyadh Military Medical City, Riyadh, Saudi Arabia. ${ }^{5}$ Department of Neurosciences, King Faisal Specialist Hospital \& Research Center, Riyadh, Saudi Arabia.

Received: 3 March 2019 Accepted: 19 June 2019

Published online: 04 July 2019

\section{References}

1. Finsterer J, Loscher W, Quasthoff S, Wanschitz J, Auer-Grumbach M, Stevanin G. Hereditary spastic paraplegias with autosomal dominant, recessive, X-linked, or maternal trait of inheritance. J Neurol Sci. 2012;318(12):1-18

2. Lo Giudice T, Lombardi F, Santorelli FM, Kawarai T, Orlacchio A. Hereditary spastic paraplegia: clinical-genetic characteristics and evolving molecular mechanisms. Exp Neurol. 2014;261:518-39.

3. Estrada-Cuzcano A, Martin S, Chamova T, Synofzik M, Timmann D, Holemans T, Andreeva A, Reichbauer J, De Rycke R, Chang DI, et al. Loss-of-function mutations in the ATP13A2/PARK9 gene cause complicated hereditary spastic paraplegia (SPG78). Brain. 2017;140(2):287-305.

4. Farazi Fard MA, Rebelo AP, Buglo E, Nemati H, Dastsooz H, Gehweiler I, Reich S, Reichbauer J, Quintans B, Ordonez-Ugalde A, et al. Truncating mutations in UBAP1 cause hereditary spastic paraplegia. Am J Hum Genet. 2019;104(4):767-73.

5. Yamamoto Y, Yoshida A, Miyazaki N, Iwasaki K, Sakisaka T. Arl6IP1 has the ability to shape the mammalian ER membrane in a reticulon-like fashion. Biochem J. 2014;458(1):69-79.

6. Akiduki S, Ikemoto MJ. Modulation of the neural glutamate transporter EAAC1 by the addicsin-interacting protein ARL6IP1. J Biol Chem. 2008; 283(46):31323-32.

7. Fowler PC, O'Sullivan NC. ER-shaping proteins are required for ER and mitochondrial network organization in motor neurons. Hum Mol Genet. 2016;25(13):2827-37.

8. Nizon M, Kury S, Pereon Y, Besnard T, Quinquis D, Boisseau P, Marsaud T, Magot A, Mussini JM, Mayrargue E, et al. ARL6IP1 mutation causes congenital insensitivity to pain, acromutilation and spastic paraplegia. Clin Genet. 2018;93(1):169-72.

9. Hu J, Shibata Y, Voss C, Shemesh T, Li Z, Coughlin M, Kozlov MM, Rapoport TA, Prinz WA. Membrane proteins of the endoplasmic reticulum induce high-curvature tubules. Science. 2008;319(5867):1247-50.

10. Novarino G, Fenstermaker AG, Zaki MS, Hofree M, Silhavy JL, Heiberg AD, Abdellateef M, Rosti B, Scott E, Mansour $L$, et al. Exome sequencing links corticospinal motor neuron disease to common neurodegenerative disorders. Science. 2014;343(6170):506-11.

11. Trujillano D, Bertoli-Avella AM, Kumar Kandaswamy K, Weiss ME, Koster J Marais A, Paknia O, Schroder R, Garcia-Aznar JM, Werber M, et al. Clinical exome sequencing: results from 2819 samples reflecting 1000 families. Eur J Human Genet. 2017;25(2):176-82.

\section{Publisher's Note}

Springer Nature remains neutral with regard to jurisdictional claims in published maps and institutional affiliations.

Ready to submit your research? Choose BMC and benefit from:

- fast, convenient online submission

- thorough peer review by experienced researchers in your field

- rapid publication on acceptance

- support for research data, including large and complex data types

- gold Open Access which fosters wider collaboration and increased citations

- maximum visibility for your research: over $100 \mathrm{M}$ website views per year

At BMC, research is always in progress.

Learn more biomedcentral.com/submissions 